\title{
Editorial
}

\section{Damage Models and Assessment Methods}

\author{
Gilbert-Rainer Gillich, ${ }^{1}$ Magd Abdel Wahab, ${ }^{2}$ Ruqiang Yan, ${ }^{3}$ and José V. Araújo dos Santos ${ }^{4}$ \\ ${ }^{1}$ Department of Mechanics, "Eftimie Murgu" University of Resita, Resita, Romania \\ ${ }^{2}$ Laboratory Soete, Faculty of Engineering and Architecture, Ghent University, Belgium \\ ${ }^{3}$ School of Instrument Science and Engineering, Southeast University, Nanjing, China \\ ${ }^{4}$ Instituto Superior Técnico, Universidade de Lisboa, Portugal
}

Correspondence should be addressed to Gilbert-Rainer Gillich; gr.gillich@uem.ro

Received 8 October 2015; Accepted 8 October 2015

Copyright (c) 2016 Gilbert-Rainer Gillich et al. This is an open access article distributed under the Creative Commons Attribution License, which permits unrestricted use, distribution, and reproduction in any medium, provided the original work is properly cited.

Damage assessment based on the vibration response of structures has been, in the last decades, an important research area in various engineering branches. It integrates knowledge from a variety of disciplines, such as mechanical and structural engineering, material science, computer science and signal processing, and data management. This special issue is dedicated to fundamental understanding of the vibrational behavior of damaged structures and to the development of methods and techniques that are able to tackle these specific problems. A number of 23 papers have been submitted, from which a total of 7 papers have been finally selected to integrate the present issue. Two among them are focused on failures in machineries: W. Wen et al. in the paper "Planetary Gearbox Fault Diagnosis Using Envelope Manifold Demodulation" extract fault characteristics from the vibration signals, since in "Undecimated Lifting Wavelet Packet Transform with Boundary Treatment for Machinery Incipient Fault Diagnosis" by L. Duan et al. a Volterra series assisted undecimated lifting wavelet packet transform is investigated for machinery incipient fault diagnosis. The presented approaches dramatically enhance the weak defect feature extraction. Damages in structural elements are the target of research presented in "Development of a Vehicle-Bridge-Soil Dynamic Interaction Model for Scour Damage Modelling" by L. Prendergast et al., "Feature Recognition for Mine Hoisting Wire Rope Based on Magnetic Flux Leakage Signals" by Z. Zhao et al., and "Experimental Study on Progressive Collapse Performance of Frame with Specially Shaped Columns Subjected to Middle Column Removal" by T. Wang et al. A model updating strategy for bolt-ball joint stiffness characterization for health monitoring is presented in "Finite Element Model Fractional Steps Updating Strategy for Spatial Lattice Structures Based on Generalized Regression Neural Network" by C. Liu et al. An interesting method to perform efficient fatigue tests is proposed by Y. Jiang et al. in the paper "Experimental Design and Validation of an Accelerated Random Vibration Fatigue Testing Methodology." They succeed to significantly reduce the test time and the sample size. The papers reflect, without doubt, significant researches and developments of this subject. We hope that readers will find all articles of the special issue useful and exciting and that the articles will stimulate further research activities in the area of damage assessment.

\section{Acknowledgments}

Acknowledgments are due to all the authors and coauthors of this special issue for their contribution.

Gilbert-Rainer Gillich Magd Abdel Wahab Ruqiang Yan

José V. Araújo dos Santos 


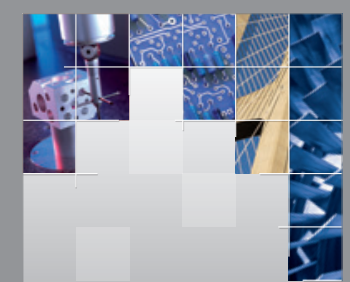

\section{Enfincering}
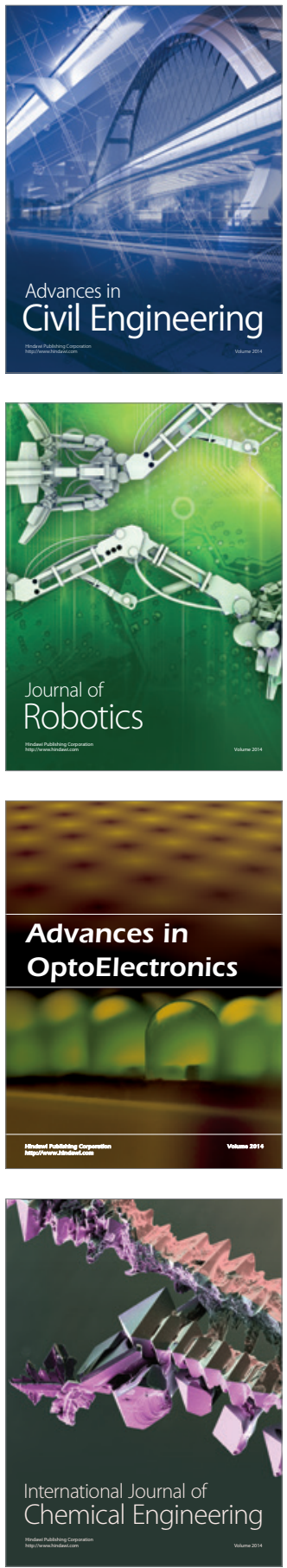

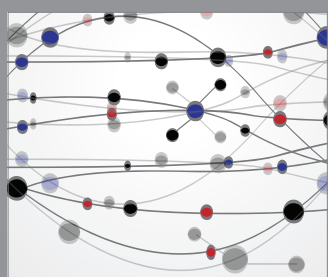

The Scientific World Journal

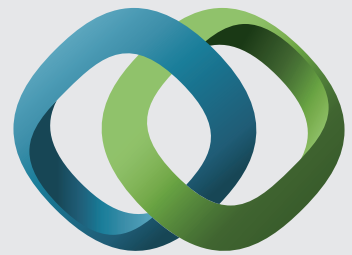

\section{Hindawi}

Submit your manuscripts at

http://www.hindawi.com
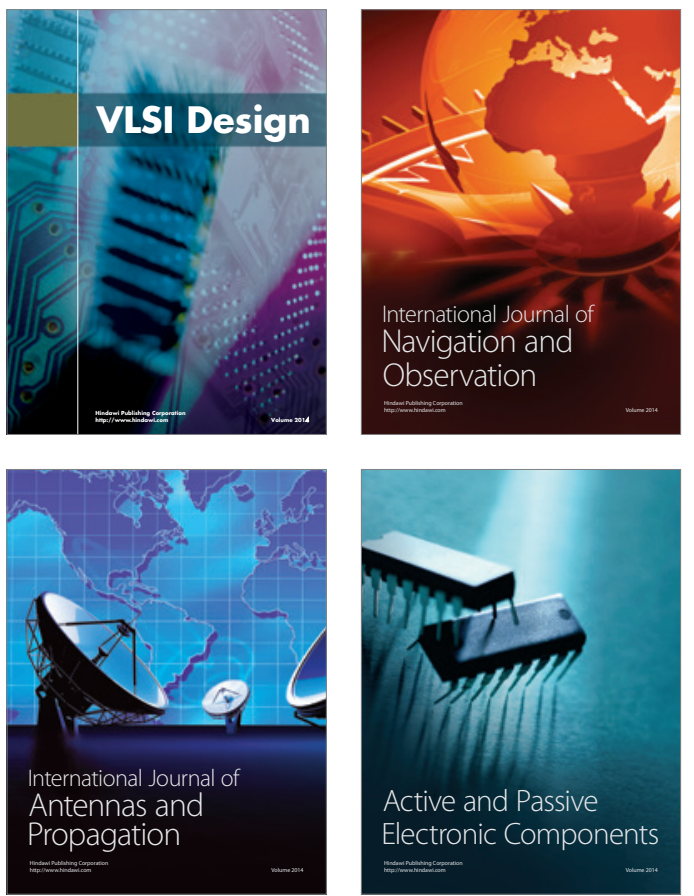
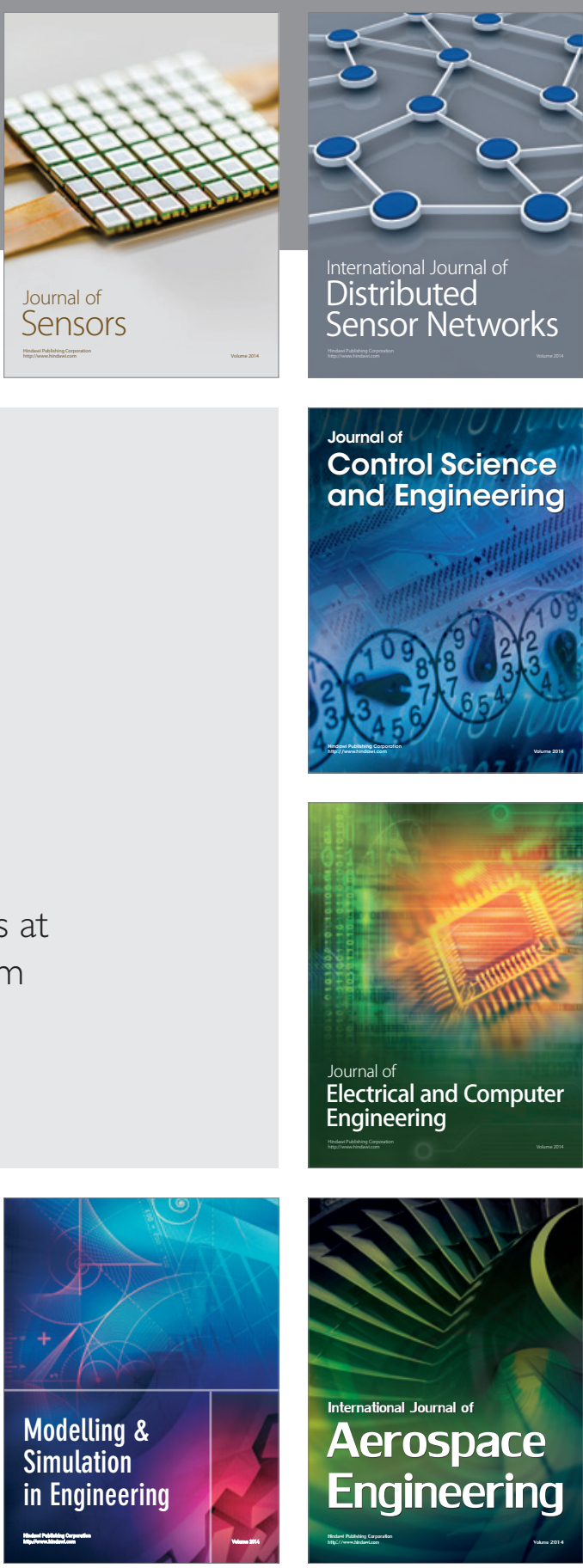

International Journal of

Distributed

Sensor Networks

Journal of

Control Science

and Engineering
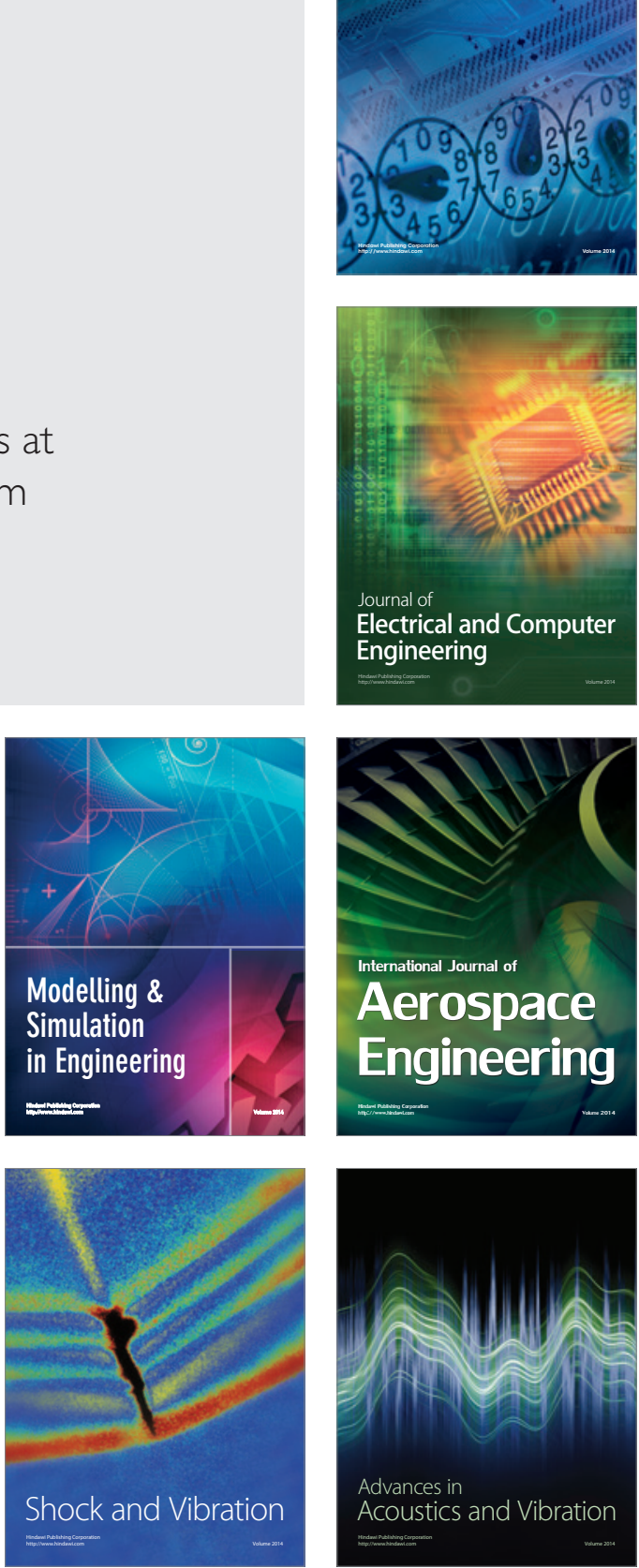